\title{
In Vivo Study of Ligament-Bone Healing after Anterior Cruciate Ligament Reconstruction Using Autologous Tendons with Mesenchymal Stem Cells Affinity Peptide Conjugated Electrospun Nanofibrous Scaffold
}

\author{
Jingxian Zhu, ${ }^{1,2}$ Xin Zhang, ${ }^{2}$ Zhenxing Shao, ${ }^{2}$ Linghui Dai, ${ }^{1} \mathrm{La} \mathrm{Li}^{2}{ }^{2}$ Xiaoqing Hu, ${ }^{2}$ \\ Xiaokun Wang, ${ }^{3}$ Chunyan Zhou, ${ }^{1}$ and Yingfang $\mathbf{A o}^{2}$ \\ ${ }^{1}$ Department of Biochemistry and Molecular Biology, Peking University School of Basic Medical Sciences, \\ 38 Xueyuan Road, Haidian District, Beijing 100191, China \\ ${ }^{2}$ Institute of Sports Medicine, Peking University Third Hospital, 49 North Garden Road, Haidian District, Beijing 100191, China \\ ${ }^{3}$ Department of Biomedical Engineering, College of Engineering, Peking University, No. 5 Yiheyuan Road, \\ Haidian District, Beijing 100871, China
}

Correspondence should be addressed to Chunyan Zhou; chunyanzhou@bjmu.edu.cn and Yingfang Ao; yingfang.ao@gmail.com

Received 22 July 2013; Revised 23 September 2013; Accepted 2 October 2013

Academic Editor: Patricia Murray

Copyright (C) 2013 Jingxian Zhu et al. This is an open access article distributed under the Creative Commons Attribution License, which permits unrestricted use, distribution, and reproduction in any medium, provided the original work is properly cited.

\begin{abstract}
Electrospinning nanofibrous scaffold was commonly used in tissue regeneration recently. Nanofibers with specific topological characteristics were reported to be able to induce osteogenic differentiation of MSCs. In this in vivo study, autologous tendon grafts with lattice-like nanofibrous scaffold wrapping at two ends of autologous tendon were used to promote early stage of ligamentbone healing after rabbit ACL reconstruction. To utilize native MSCs from bone marrow, an MSCs specific affinity peptide E7 was conjugated to nanofibrous meshes. After 3 months, H-E assessment and specific staining of collagen type I, II, and III showed direct ligament-bone insertion with typical four zones (bone, calcified fibrocartilage, fibrocartilage, and ligament) in bioactive scaffold reconstruction group. Diameters of bone tunnel were smaller in nanofibrous scaffold conjugated E7 peptide group than those in control group. The failure load of substitution complex also indicated a stronger ligament-bone insertion healing using bioactive scaffold. In conclusion, lattice-like nanofibrous scaffold with specific MSCs affinity peptide has great potential in promoting early stage of ligament-bone healing after ACL reconstruction.
\end{abstract}

\section{Introduction}

Tissue engineering technique emerges as a new tool in tissue regeneration these years. Nanosized material was frequently used as scaffold in tissue engineering because of its superior characteristics like huge surface area and superior biological compatibility. Nanofibrous scaffold fabricated by electrospinning technique has been used in bone, neuron, and ligament regeneration. We developed a specific nanotopographical electrospun scaffold which could induce osteogenesis of rat bone marrow derived mesenchymal stem cells (MSCs) in vitro. And now we want to testify the ability of this scaffold in accelerating bone formation at the ligament-bone insertion sites after rabbit ACL reconstruction.
Nanofibrous scaffolds are appropriated for cell adhesion and growing because of the huge surface area and fibers in nanoscale which can mimic natural extracellular matrix $[1,2]$. Among the methods of fabricating nanofibrous scaffolds, electrospinning is the most widely used given its simplicity and efficiency. However, mechanical properties of electrospun meshes render it impossible to be a substitution for ACL reconstruction alone. Therefore some researchers designed composite scaffold combining firm artificial material with nanofibrous surface made by electrospinning [3, 4]. But autologous tendons especially hamstring tendons, which were commonly used in clinical surgery, were still verified to be better than artificial scaffolds for their good biocompatibility 
and mechanical properties $[5,6]$. So combining autologous tendon and electrospun meshes to form a new scaffold should satisfy both the mechanical and cell growth requirements.

MSCs have been widely used in tissue engineering studies and have been proved to have great potential in clinical application because of their convenience in isolation, lack of significant immunogenicity, high capacity of expansion, and potential to differentiate into tissue-specific cell types [7-9]. However MSCs should be first homed and entrapped into the site of tissue damage so as to allow MSCs to properly perform their functions during the whole repair process [10]. In our previous research we screened an affinity peptide of bone marrow-derived MSCs using phage display technique to solve the problem of low efficiency and engraftment of MSCs in tissue engineering [11]. As a result, covalently conjugating the peptide E7 on the synthetic electrospun mesh significantly enhanced the MSC recruitment of scaffold in vivo. Therefore we used this technique for the same purpose in our study.

As new bone formation is of vital importance for ligament-bone healing after ACL reconstruction, many composite scaffolds were designed to promote osteogenesis of MSCs at the insertion site. In bone tissue engineering, composition mimic native extracellular matrix like hydroxyapatite, collagen, chitosan, and so forth was added to promote osteogenic differentiation of MSCs [12,13]. Also, bioactive cytokines like BMP2 were used as chemical inductive agents, and mechanical stimulation can promote osteogenic differentiation of MSCs as well [14]. In recent years, topological characteristics of nanofibrous scaffold was confirmed to be an important factor in deciding the destiny of cell growing on them. Specific topographical scaffold can promote cell adhesion, migration, proliferation, and differentiation $[15$, 16]. In our previous research, we found that the latticelike nanofibrous mesh can promote osteogenesis of rat MSCs by regulating integrin b1 (ITGB1), ras homolog gene family member A (RhoA)/Rho-associated protein kinase (ROCK), extracellular regulated protein kinases (ERK), and runt related transcription factor 2 (Runx2) expression [17]. However, few studies were available that have tested the osteoinductive ability of electrospun meshes in vivo. In this study, we designed a new scaffold, which is combined latticelike nanofibrous meshes with bone marrow MSCs affinity peptide, and was used in rabbit ACL reconstruction model together with autologous hamstring tendon. The aim of this study is to compare the osteogenic ability of tendon grafts with or without nanofibrous scaffolds and to explore the potential application of electrospun scaffolds in ligament tissue engineering.

\section{Methods and Materials}

2.1. Electrospinning PLLA Nanofibrous Scaffold. Lattice-like patterned poly-L-lactide (PLLA) nanofibrous scaffolds were fabricated as described in our previous research. The polymer solution was prepared by dissolving $1.0 \mathrm{~g}$ of PLLA powders in $10 \mathrm{~mL}$ tetrafluoroethylene and stirred overnight. The solution was electrospun from a $20 \mathrm{~mL}$ syringe with a steel needle (inner diameter of $0.5 \mathrm{~mm}$ ) at a rate of $0.5 \mathrm{~mL} / \mathrm{h}$ continuously with a programmable syringe pump (Top 5300, Japan). A voltage $(12 \mathrm{kV})$ was applied to the tip of the needle by the use of a high-voltage supply (DW-P303-1AC, China) when a fluid jet was ejecting. To obtain patterned PLLA nanofibrous scaffolds, a copper wire grid $(r=10 \mathrm{~cm})$ was used as a collector at a distance of $15 \mathrm{~cm}$ from the tip of the needle. Before using, all scaffolds were exposed to a vacuum oven (DZF-6210, Bluepard, China) at room temperature for 2 weeks to remove residual solvent.

\subsection{MSCs Affinity Peptide E7 Biopanning and Conjugation.} The bone marrow MSCs of Wistar rats were harvested using standard protocol in our lab. MSCs were derived from two lower extremities of rats with the approval of the Animal Ethics Committee of Peking University Third Hospital. MSCs specific affinity peptide was biopanning by the phage display technique following a previously described procedure with modifications $[11,18]$. In brief, first a peptide phage display library was commercially constructed (Ph.D.-7 phage display library, NEB, Beverly, MA, USA) and incubated with MSCs, synovial cells were used as negative control. Then the phage clones binding MSCs were amplified, titrated and, purified according to the manufacturer's standard protocol. Three to four rounds of selection were performed until the optimal results were obtained. The experiments were duplicated for verification. A specific peptide was identified in the MSCaffinity clones and designated as E7. The peptides were dissolved in DMSO to yield a $2 \mathrm{mg} / \mathrm{mL}$ concentration before usage.

The PLLA meshes were immersed into a $10 \% \mathrm{w} / \mathrm{v}$ solution of 1,6-hexanediamine (Sigma, St. Louis, MO, USA) prepared in isopropanol at $37^{\circ} \mathrm{C}$ for $1 \mathrm{~h}$. After the exposure, the PLLA meshes were thoroughly washed in ultrapure water and dried under vacuum at room temperature. For the conjugation of E7 peptides to the surface of meshes, the aminated PLLA meshes were washed thrice with activation buffer $(0.1 \mathrm{M}$ PBS containing $0.15 \mathrm{M} \mathrm{NaCl}, \mathrm{pH} 7.2)$ before treatment, and sulfosuccinimidyl-4- (N-maleimidomethyl) cyclohexane-1carboxylate (sulfo-SMCC) (Thermo Fisher Scientific Inc., Rockford, IL, USA) was used as a cross-linker. A total of $500 \mathrm{~mL}(2 \mathrm{mg} / \mathrm{mL})$ sulfo-SMCC solution was added onto the aminated PLLA meshes, incubated for $1 \mathrm{~h}$ at room temperature, and washed with conjugation buffer (activation buffer containing $0.1 \mathrm{M}$ EDTA, $\mathrm{pH}$ 7.0). The peptides were dissolved in conjugation buffer at a concentration of $0.1 \mathrm{mM}$. Up to $500 \mathrm{~mL}$ of the peptide solution was applied onto the sulfo-SMCC-treated PLLA scaffold and incubated overnight at $4^{\circ} \mathrm{C}$. The peptide conjugated PLLA meshes were thoroughly washed thrice with ultrapure water and dried under vacuum at room temperature. The whole mesh was cut into $1 \mathrm{~cm}^{2}$, sterilized by immersing in $75 \%$ ethanol over-night, washed thrice with ultrapure water, and immersed in PBS over $4 \mathrm{~h}$. Before implantation, the meshes were kept in $4^{\circ} \mathrm{C}$ refrigerator.

2.3. MSCs Attachment on Different Scaffolds. The MSCs at passage 3 were seeded onto the blank or E7-conjugated lattice PLLA meshes. After $24 \mathrm{~h}$ of incubation, the PLLA meshes were washed from three to five times with PBS 
and fixed with $4 \%$ paraformaldehyde for $10 \mathrm{~min}$ at room temperature. Cells were then preincubated in $100 \mu \mathrm{L}$ of primary blocking solution (PBS, 1\%BSA, and 0.1\% NP-40) for $20 \mathrm{~min}$ and then incubated with $10^{-7} \mathrm{M}$ Alexa Fluor 488 phalloidin conjugate (Molecular Probes) for $1 \mathrm{~h}$. After three washes in PBS, nuclei were stained with $10 \mu \mathrm{g} / \mathrm{mL}$ Hochest 33342 at room temperature for $30 \mathrm{~min}$. Mounting medium (35 $\mu \mathrm{L}$, Zhongshan) was dispensed onto the cells, and a glass coverslip was placed on the slide before placing it in the freezer prior to observation. Actin structures were examined by laser confocal scanning microscopy (FV 1000 Olympus IX81). Cell attachment was observed by confocal microscope at both ridge regions and nonwoven regions of lattice. And percentage of cell attachment to material within each group were measured using Image-Pro Plus 6.0 software (Media Cybernetics, Silver Spring, MD, USA) by 5 random areas were used in one photograph.

2.4. ACL Reconstruction Surgery. Animal experiments were approved by Ethical Committee of third hospital, Peking University. Forty-eight New Zealand white rabbits (12 weeks old, $2.5-3.0 \mathrm{~kg}$ ) were divided into three groups: BIO group (lattice-like PLLA scaffold with MSCs affinity peptide; $n$ 16), PLLA group (lattice-like PLLA scaffold alone; n 16), and control group with single reconstruction surgery. Hamstring tendon grafts were harvested from both two knees of each rabbit. Then two tendons were bound by braiding two ends with 3-0 braided nylon thread. Nanofibrous meshes were braiding together with tendon in BIO or PLLA reconstruction groups at this time. The right knee joint of each rabbit was exposed using a midline skin incision, and lateral parapatellar arthrotomy was performed. The patella was dislocated totally with the knee extended. With the knee placed in its hyperflexive state, the original ACL was removed by sharp dissection of the tibial and femoral attachment sites, while the fat pad was left intact. Using a $2.0 \mathrm{~mm}$ diameter Kirschner wire and a drill, bone tunnels were made from the ACL femur attachment sites to the lateral femoral condyles, as well as from the ACL tibial attachment sites to the medial tibia. The tibial sites were distal and lateral to the tibial tubercles. After placing the grafts into two bone tunnels, both two ends were fixed by bone bridge (Figures 3(a)$3(\mathrm{f})$ ). This was done with the knee at $45^{\circ}$ of flexion, while the constructs were manually exposed to maximum tension. Penicillin (200000 U/kg) was subcutaneously administered to prevent postoperative infection. Enhanced antibiotic was used for 3 days after surgery. At 1 or 3 months after surgery, the knee joints were harvested for histological and mechanical examinations. At the specified times, rabbits were sacrificed by intravenous air embolism.

2.5. Measurement of Bone Tunnel by CT Scanning. All 48 hind limbs were harvest after rabbit was sacrificed immediately. Whole limb was scanned by 64-row CT scanner (LightSpeed VCT; GE Medical System, Chalfont St Giles, UK) using the same parameter of human hands scanning. Both the femoral and tibial tunnels were measured by one and the same technician, to ensure no operating errors. The cross-section of bone tunnel was determined by 3 steps: first step, confirm the middle of articular according the patellar position; then draw the long axis of bone tunnel in femora or tibia by adjusting the sagittal and coronal plane to show the longest tunnel; at last the cross sectional images were taken perpendicular to the long axis of bone tunnel (Figure 4). All slices of each sample were scanned and the minimal diameter of bone tunnel could be calculated by 3-dimensional reconstruction. All data were analyzed by 3 -different researchers and both femoral and tibial tunnel diameter was taken as one specimen.

2.6. Histological and Immunohistological Assessment. Eight of specimens in each group were prepared for histological assessment. Ligament-bone insertion was harvested by cutting from sagittal plane $1 \mathrm{~cm}$ away articular. After all soft tissue removing, transplanted ligament was cut off in the middle part, fixed in formalin (4\% formalin in phosphatebuffered saline), and embedded in paraffin. Six micron thick tissue sections were cut from these paraffin blocks. All of the specimens were stained with hematoxylin and eosin $(\mathrm{H}-$ E), sirius red, and immunohistochemical staining to display the histological characteristics and distribution of collagen type I, II, and III. Distribution of collagen type I and III was displayed by sirius red staining and observed by polarized light microscope (Laica DM2500, Wetzlar, Germany). Distribution of collagen II was evaluated with a specific antibody to rabbit collagen type II (Calbiochem, Darmstadt, Germany). Before staining, the samples were treated with pepsin working solution (ZhongShanJinQiao, Beijing, China) for $15 \mathrm{~min}$ and then incubated with primary antibody overnight. Following PBS washing, biotinylated secondary antibodies were added for 1 hour at $37^{\circ} \mathrm{C}$, then the samples were colorated with 3,3-diaminobenzidine (DAB). Each specimen was viewed by light microscopy (Olympus BH-2, Tokyo, Japan) and photographs were taken from 3 randomly chosen representative microscopic fields at a magnification of 40200x.

2.7. Biomechanical Test of Substitution Complex. After CT scanning, the lower extremities of another 8 animals in each group were disarticulated at the hip joint and stored at $-80^{\circ} \mathrm{C}$ refrigerator. Before mechanical testing, each knee was thawed overnight at $4^{\circ} \mathrm{C}$ and all soft tissues around the knee were carefully removed except the ACL. The prepared femur-ACLtibia complex was then mounted onto a conventional tensile tester. The femur and the tibia were fixed in cupreous holders using 2 Kirschner wires. The knee was fixed at $45^{\circ}$ of flexion with maximal tensile resistance during testing. The specimen was cycled between 0 and $0.5 \mathrm{~mm}$ deformation 10 times at a rate of $5 \mathrm{~mm} / \mathrm{min}$ for pretension. Subsequently, the specimen was stretched to failure at a rate of $0.1 \mathrm{~mm} / \mathrm{s}$. The loaddeformation curve was recorded and the ultimate load was then obtained. The linear stiffness was defined as the bestfitting straight line drawn through the linear portion of the load-deformation curve. The specimens were kept moist at 
all times during preparation and testing with normal saline solution.

2.8. Statistical Analysis. All data were shown as the mean with standard deviation. One-way analysis of variance was performed when comparing data of 3 groups. Other statistical comparisons were performed with the paired-samples $t$-test. SPSS 14.0 software was used for statistical calculation. The significance level was set at $P<0.05$.

\section{Results}

3.1. Lattice-Like PLLA Nanofibrous Meshes Synthesis and Observation. Propelled by electrostatic forces, lattice-like fibrous PLLA meshes could be obtained by using copper wire grid as collector (Figure 1(a)). The lattice-like mesh macroscopically resembled gauzes and followed the pattern of grid (Figure 1(b)). Detailed structure of nanofibers in the scaffolds was observed by scanning electron microscopy (Figures 1(c), $1(\mathrm{e})$, and $1(\mathrm{f}))$. Fibers depositing on the ridge regions were parallel-aligned (Figure 1(e)) while in those regions between ridges were randomly distributed (Figure $1(\mathrm{f})$ ). The diameter of nano fibers ranged from 200 to $1200 \mathrm{~nm}$ (Figure 1(e)).

3.2. MSCs Attachment on PLLA Nanofibrous Meshes. The attachment of MSCs on scaffolds with or without affinity peptide was observed by laser scanning confocal microscope. Staining of cytoskeleton and nuclei of MSCs showed well attaching and spreading of cells on both two kinds of scaffolds. But the cell number was obviously different on E7 conjugated PLLA meshes at different regions of lattice meshes (Figures 2(a) and 2(c)). Cell morphology of MSCs growing on E7 conjugated PLLA meshes appeared better organized and was more compliance with the nanofiber distribution than on PLLA meshes (Figures 2(b) and 2(d)). Percentage of cell attachment to different kinds of scaffolds was obviously different, which was $89.72 \pm 1.66 \%$ and $60.24 \pm 10.42 \%(P<$ $0.05)$ on PLLA meshes with or without E7 peptide.

3.3. Gross Observation. Twenty-four rabbits were sacrificed at 1 or 3 months after ACL reconstruction surgery, respectively. Gross morphology of knee joint was compared after operation between three groups. At 12 weeks the regenerated ligament showed a stable fixation in all three groups without any ends pulling out of tunnels. There was not obvious infection in joint cavity; meanwhile synovial fluid was clear in three groups. But all joints exhibited a little degeneration with cartilage injury, to some extent, which might because of the damage in surgery procedure or instability after reconstruction of ACL. The gross findings revealed that reconstructed ligament was wrapped by synovial membrane, but two bundles of tendon were still distinguishable (Figures $3(\mathrm{~g})-3(\mathrm{i}))$.

3.4. Bone Tunnel Measurement. The cross-sectional images perpendicular to the longitudinal axis of bone tunnel were reconstructed in high-resolution CT. The newly formed mineralized tissue could be examined by screening all slices of
TABLE 1: Bone tunnel measured by CT and failure load of grafts.

\begin{tabular}{lccc}
\hline \multirow{2}{*}{ Group } & \multicolumn{2}{c}{ Bone tunnel $(\mathrm{mm})$} & Failure load $(\mathrm{N})$ \\
& $1 \mathrm{M}$ & $3 \mathrm{M}$ & $3 \mathrm{M}$ \\
\hline REC & $7.50 \pm 1.80$ & $10.16 \pm 2.62^{*}$ & $34.58 \pm 4.08$ \\
PLLA & $5.96 \pm 0.90$ & $6.72 \pm 1.66$ & $82.59 \pm 3.33^{*}$ \\
BIO & $5.32 \pm 2.45$ & $4.20 \pm 1.87$ & $141.50 \pm 3.90^{*}$ \\
\hline
\end{tabular}

REC: reconstruction group, PLLA: reconstruction with PLLA scaffold, and BIO: reconstruction with E7 peptide conjugated PLLA scaffold. Student's test was used and significance of ${ }^{*} P<0.05$.

each sample and the minimal diameter of bone tunnel could be calculated. In both control group and PLLA reconstruction group, the bone tunnel was larger at 3 months after surgery than 1 month, which was known as the "enlargement of bone tunnel." Though in BIO reconstruction group there was no obvious enlargement at 3 months, bone tunnel was smaller than the other two groups both at 1 and 3 months, which was $5.32 \pm 2.45$ and $4.20 \pm 1.87 \mathrm{~mm}$, respectively (Table 1 ). The consecutive CT images showed that there was no obvious mineralized tissue formed in tibial and femoral bone tunnels after 4 and 12 weeks in all groups (Figures 4(b) and 4(d)). After CT scan, the biomechanical test or histological staining was performed to further examine the changes of ligamentbone insertion.

3.5. Biomechanical Analysis. Four specimens in each group at 3 months after surgery were tested to evaluate tensional properties of the reconstructed ACL. Results showed that all substitution complex in $\mathrm{BIO}$ reconstruction group were broken in the midpart of regenerated ligaments during mechanical test. Two of four specimens in PLLA group were pulled out of ligament insertion sites, while in control group there was one. The load-deformation curves changed smoothly from a flat slope to a steep one with extension of implants and suddenly fell at failure load. The control group recorded a maximum tensile load of $34.58 \pm 4.08 \mathrm{~N}$, while the PLLA reconstruction group had a failure load of 82.59 $\pm 3.33 \mathrm{~N}$. The failure load in BIO reconstruction group was $141.50 \pm 3.90 \mathrm{~N}$ at 12 weeks postoperatively, which was also significantly higher than that of control $(P<0.05)$ (Table 1$)$.

3.6. Histological Assessment of Ligament-Bone Insertion. Histological observation demonstrated that the fibrovascular connective tissue, fibrocartilage, and the Sharpey's fibers were gradually developed between the implants and bone tunnel in BIO reconstruction group. At 4 weeks postoperatively, only a thin layer of fibrovascular connective tissue was observed on the interface of regenerated ligament in control and PLLA reconstruction groups (Figures 5(a) and 5(b)). But in $\mathrm{BIO}$ reconstruction group the implant-bone interface was filled with loose fibrocartilage tissue with a few chondrocytes scattered between bone and regenerated ligament (Figure 5(c)). After 12 weeks, the thin layer of connective tissue became much denser in PLLA reconstruction group (Figure 5(e)) while in control group, the more organized perpendicular collagen fibers resembling the Sharpey's fibers were observed to form the indirect junction between ligament and bone 


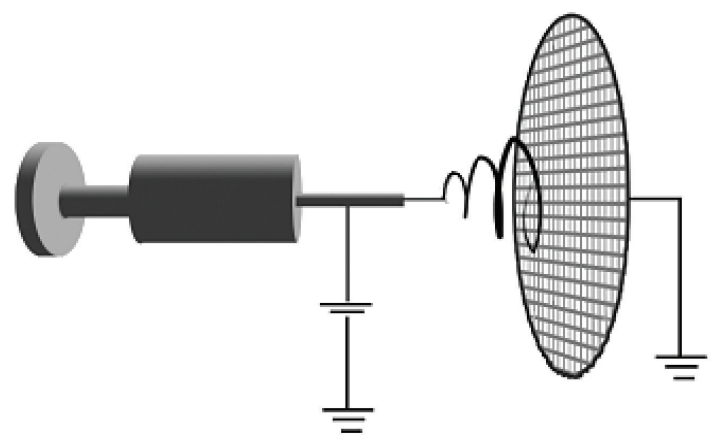

(a)

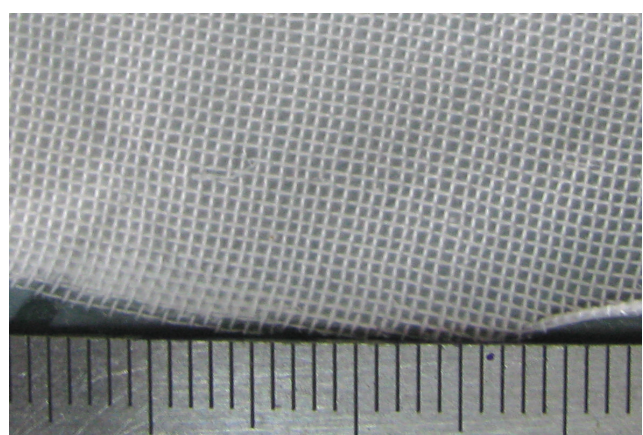

(b)

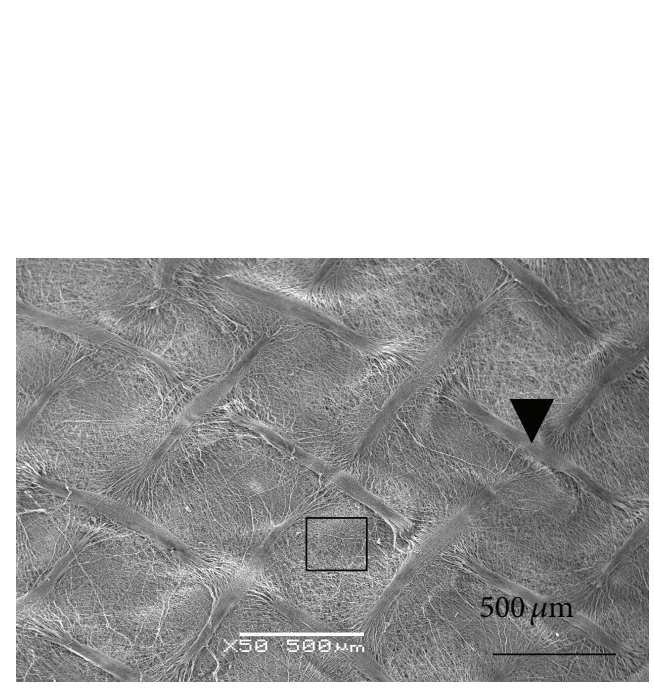

(c)

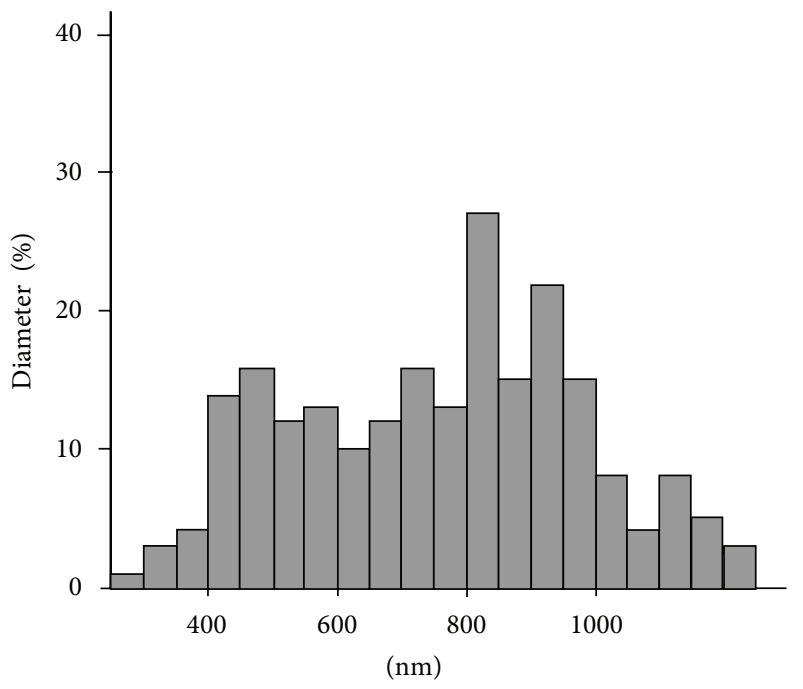

(d)

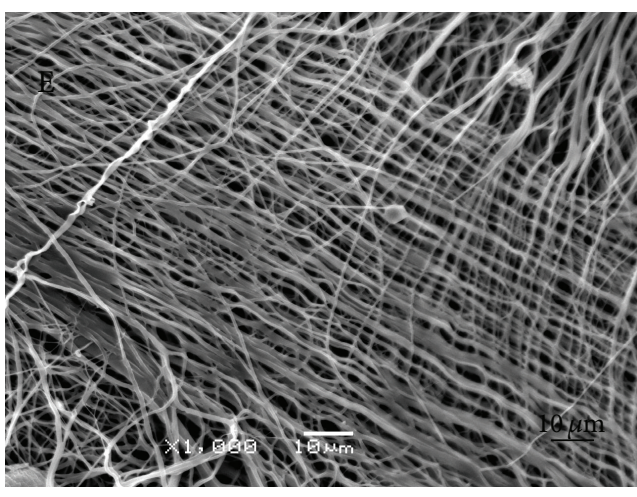

(e)

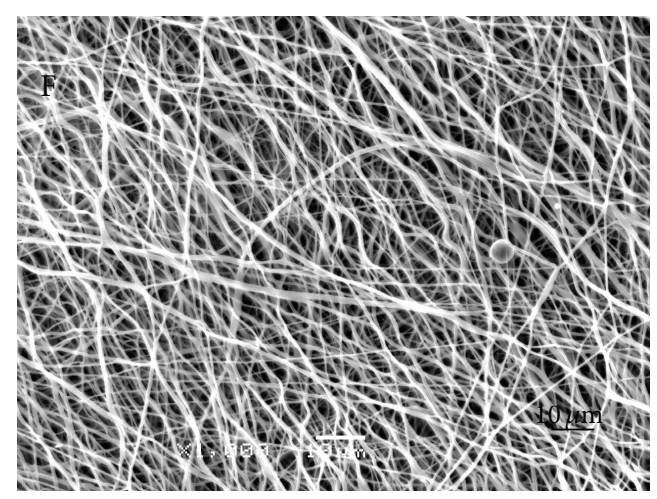

(f)

FIGURE 1: Schematic illustration of electrospinning devices and scanning electron microscope observation of nanofibers characteristics in lattice-like electrospun scaffolds. (a) Schematic diagram of electrospun scaffold device showed lattice-like fibers collecting by copper wire grid. (b) Picture of scaffold showed general view of lattice-like PLLA meshes. ((c), (e), and (f)) Micrographs showed nanofibers distribution in different regions of lattice-like PLLA meshes. Detailed view showed local enlarged fibers on ridge (e) and nonwoven region (f) of each lattice. (d) Histogram showed distribution of nanofibers' diameter in scaffolds.

at 12 weeks (Figure $5(\mathrm{~d})$ ). At the same time, fibrocartilage appeared with tide line mark between ligament and bone in BIO reconstruction group. A gradual transition from bone, mineralized fibrocartilage and fibrocartilage, into the tendon substance was successfully reconstructed (Figure 5(f)).

At 3 months after surgery, collagen type II, I, and III distribution of insertion site was detected by immunohistochemical and Sirius red staining, respectively. Results indicated that a large amount of collagen fibers making up of type III and I grow from ligament to bone in control group (Figure 6(a)), while little mature cartilage tissue was found at insertion site in this group by collagen II staining (Figure 6(d)). In PLLA and BIO reconstruction groups, collagen III amount decreased at insertion site while collagen I increased (Figures 6(b) and 6(c)). Meanwhile fibrocartilage with aligned chondrocytes was found in BIO group 


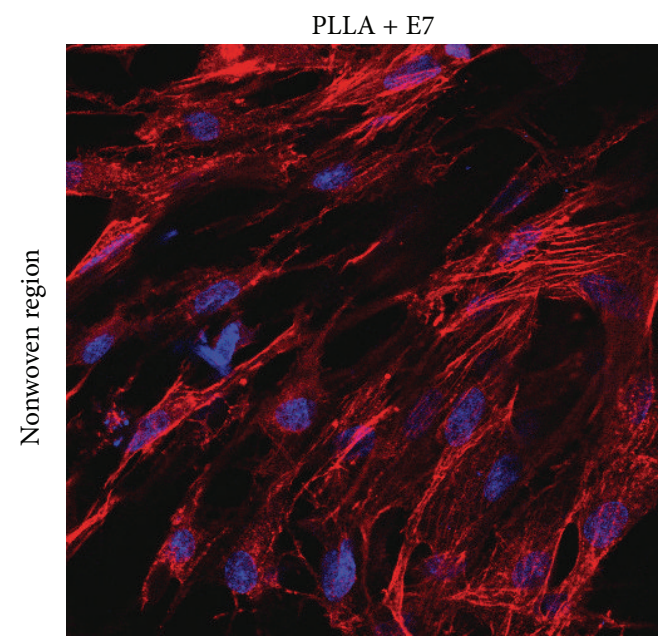

(a)

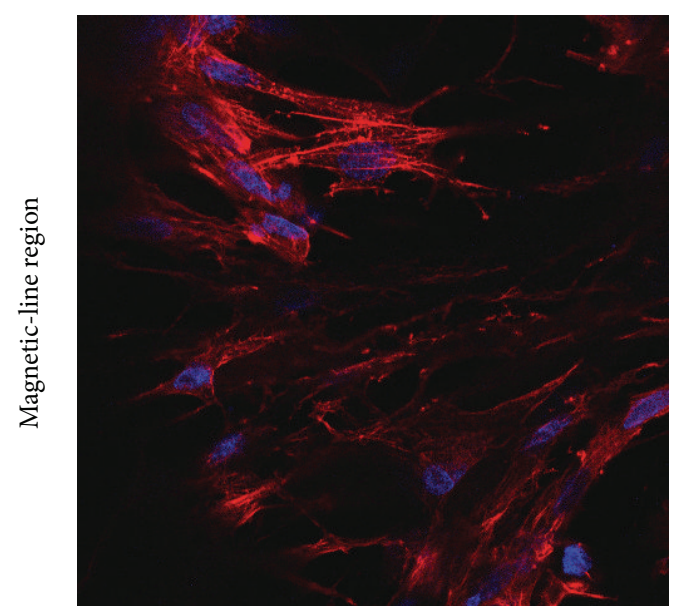

(c)

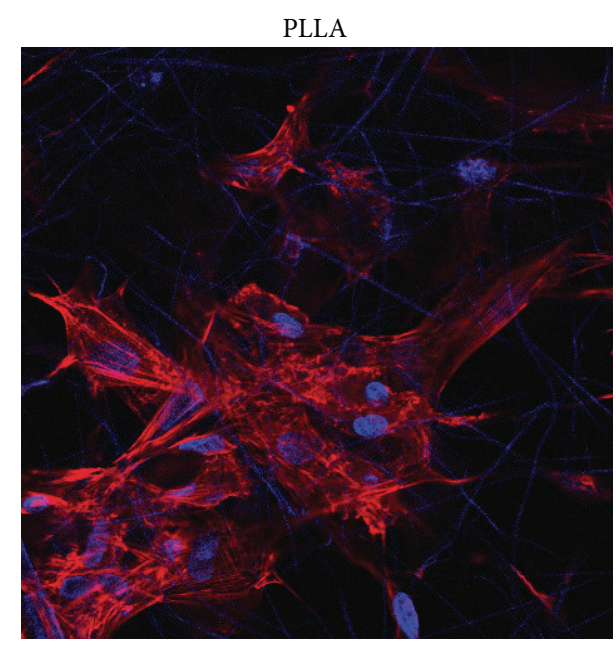

(b)

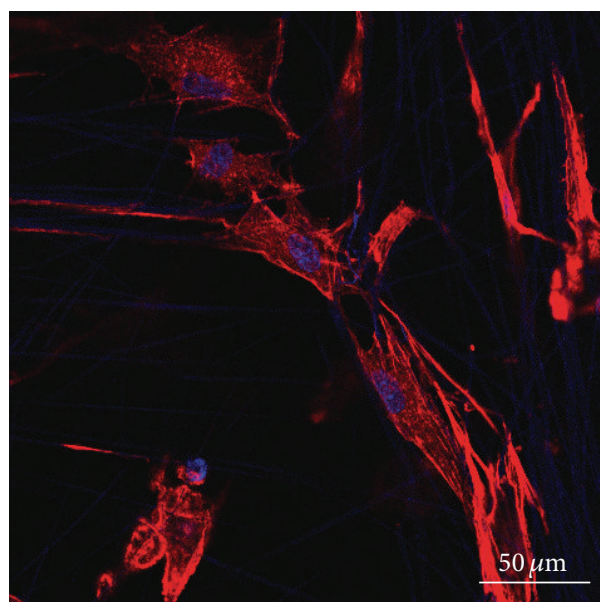

(d)

FIGURE 2: Laser scanning confocal microscopy image showed attachment of MSCs on different scaffolds. Morphology of MSCs was displayed by F-actin (red) and nuclei (blue). Cell attachment and spreading on E7 conjugated PLLA meshes were shown in both nonwoven and near ridge regions of lattice ((a), (c)). MSCs that grew on PLLA meshes were shown as control ((b), (d)).

by immunohistochemical staining (Figure 6(f)). In PLLA group, few collagen II fibers and chondrocytes indicated an immature insertion site (Figure 6(e)).

\section{Discussion}

The ligament-bone insertion is important for the effective transmission of mechanical force and the reduction in stress gradient between the soft ligament and hard bone tissue. The healing of ligament-bone insertion after ACL reconstruction is of vital importance for the stability of implantation especially at early stage. Many kinds of approaches like cytokines, biomaterials, biological and chemistry reagents, stem cells, and genes were added to strengthen the healing of ligament-bone insertion [19-23]. Their objectives were the same, which is to promote local bone formation. While drilling bone tunnel, many bone marrow MSCs and cytokines were released and leaked out into the articular cavity. Using these mixture would be more convenient and effective than using extrinsic MSCs supplement. In our study, nanofibrous meshes which wrapped two ends of tendon graft can fill the interspace between tendon and bone tunnel and increase the contact area to absorb the leaking MSCs from bone marrow. Meanwhile our results indicated higher adhesion level of MSCs on nanofibrous meshes conjugated with E7 peptide which was proved to have specific affinity to MSCs. The specific affinity peptide together with physical absorption ability of nanofibrous scaffold promoted the utilization of endogenous bone marrow MSCs.

The ultimate goal in the design of biomimetic materials for use in tissue engineering is to generate biocompatible scaffolds with appropriate mechanical and chemical properties. Recent efforts have therefore been focused on the construction and modification of surfaces targeted to support tissuespecific cell functions including adhesion, growth, differentiation, motility, and the expression of tissue-specific genes [24]. There are three types of modification of biomimetic surfaces to mimic natural ECM: physical properties, chemical signals, and the nanoscale topography of microenvironmental adhesive sites. Recently, the development of new 


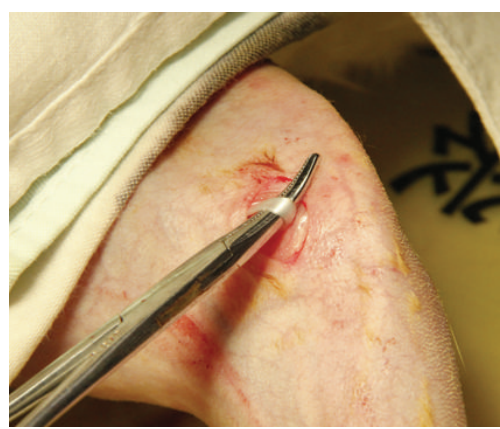

(a)

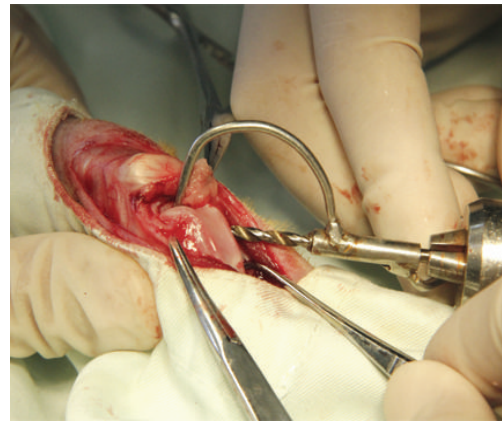

(d)

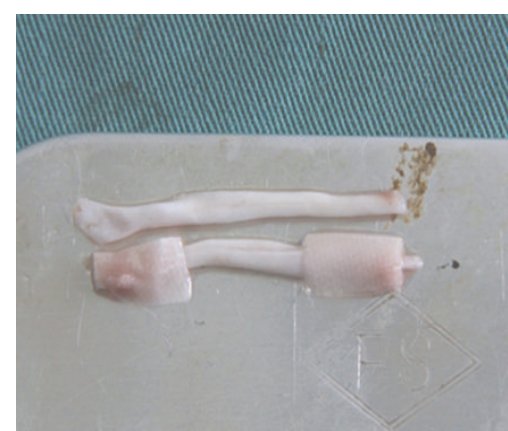

(b)

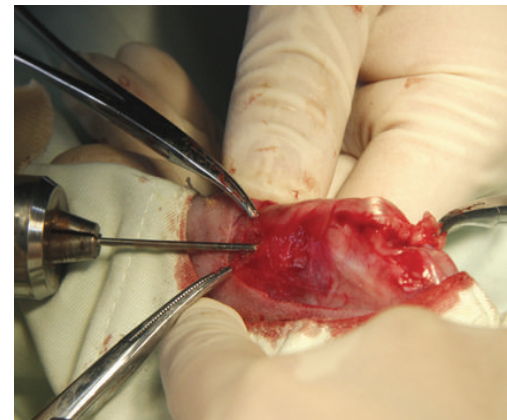

(e)

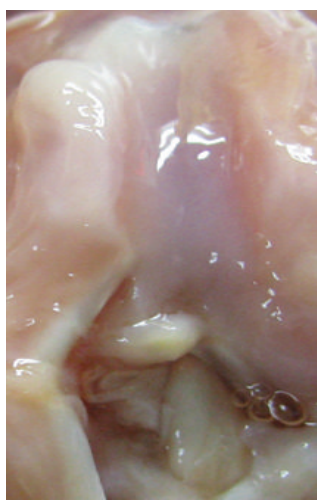

PLLA-R

(h)

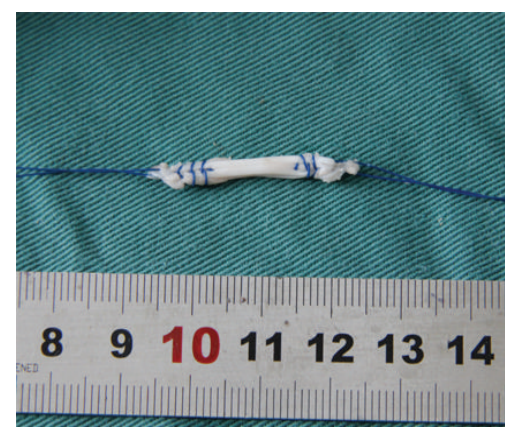

(c)

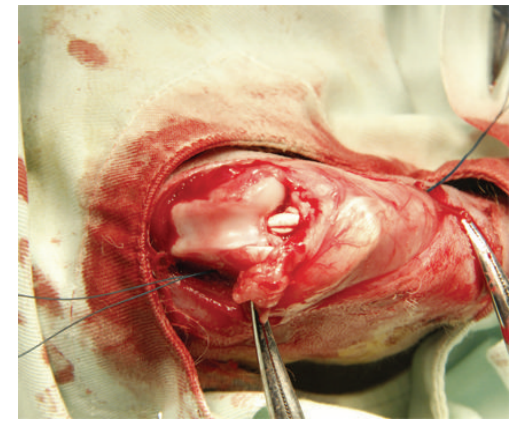

(f)

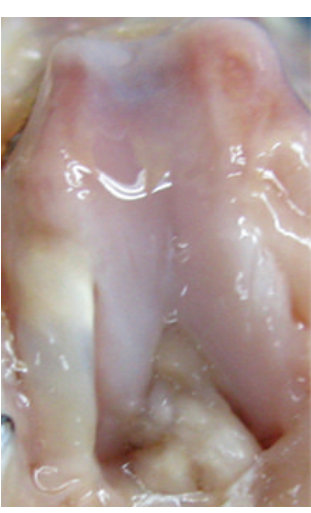

Reconstruction

(g)

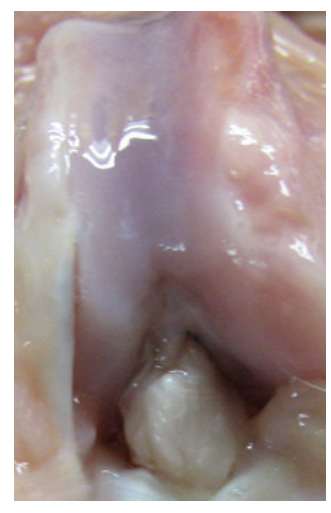

BIO-R

(i)

FIGURE 3: Pictures showing rabbit ACL reconstruction surgery procedure and gross morphology of reconstructed ACL at 3 months after surgery. Hamstring tendon grafts were harvested from both two knees of each rabbit (a). PLLA scaffold or PLLA scaffold with E7 peptide conjugation was wrapped at two ends of tendons in PLLA reconstruction group and BIO reconstruction group (b) and braided with nylon thread (c). The right knee joint of each rabbit was exposed and original ACL was removed. Bone tunnels were made from the ACL femur attachment sites to the lateral femoral condyles (d), as well as from the ACL tibial attachment sites to the medial tibia (e). After placing the transplants into two bone tunnels, both two ends were fixed by bone bridge (f). At 90 degree flexion of knee joint, both reconstructed ACL and cartilage of femoral condyle could be exposed in control group (g), PLLA reconstruction group (h), and BIO reconstruction group (i).

nanotechnologies allowing the construction of a geometrically defined microenvironment for cells at the nanoscale could induce specifically differentiation of MSCs. In others' researches, aligned nanofibrous scaffolds can induce MSCs to differentiate into ligament, tendon fibroblasts, or fibrochondrocytes [25-27]. In our and others' research, lattice-like nanofibrous meshes can induce osteogenic differentiation of MSCs, which is more important in ligament-bone healing, through integrin signaling in vitro [28]. So we testified it by these in vivo experiments. Histological staining indicated a fibrous tissue connection of ligament to bone in control group with tendon grafts only, while in PLLA scaffold groups with or without peptide there was fibrocartilage formation at different levels. This was confirmed by specific stain of collagen type I, II, and III. It has been known that normal ligament and bone were mainly composed of type I collagen while repaired scar tissue was mainly made up of type III collagen. In control group, the insertion site was filled with disorderly arranged collagen III and I which formed an indirect junction. That coincided with early stage healing of 


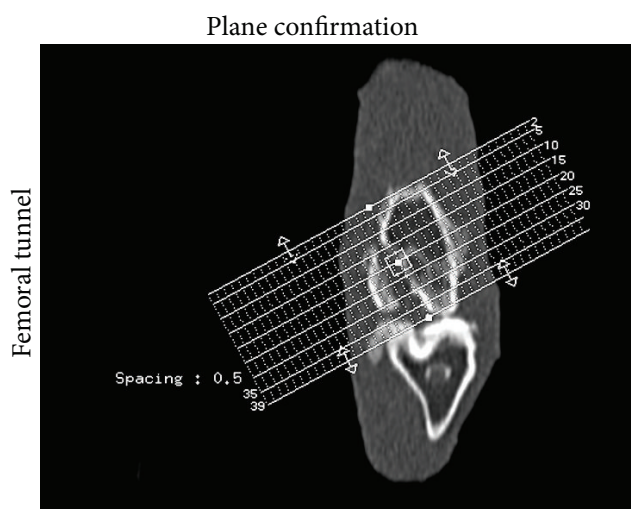

(a)

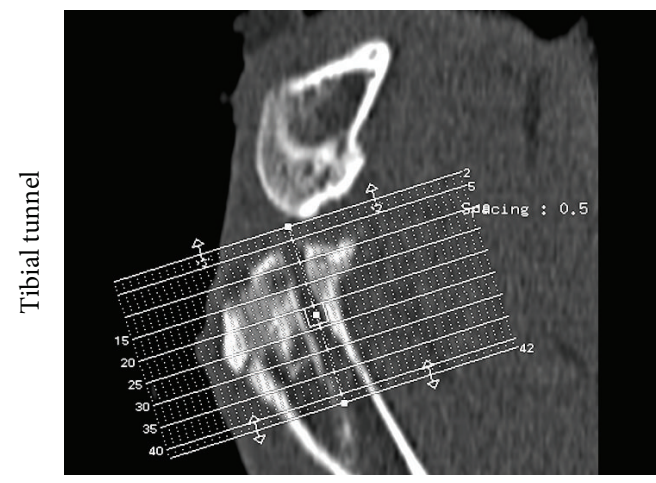

(c)
Diameter measurement

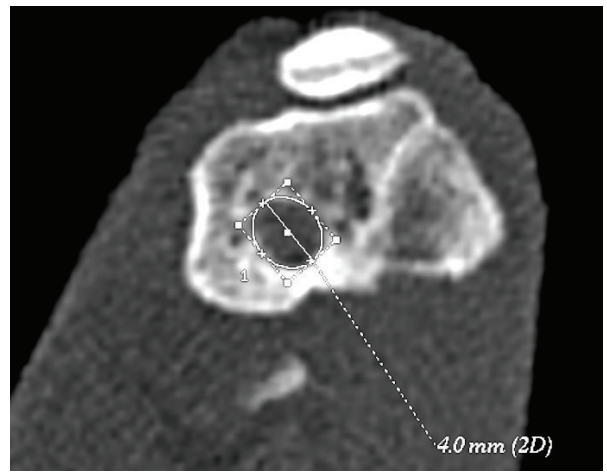

(b)

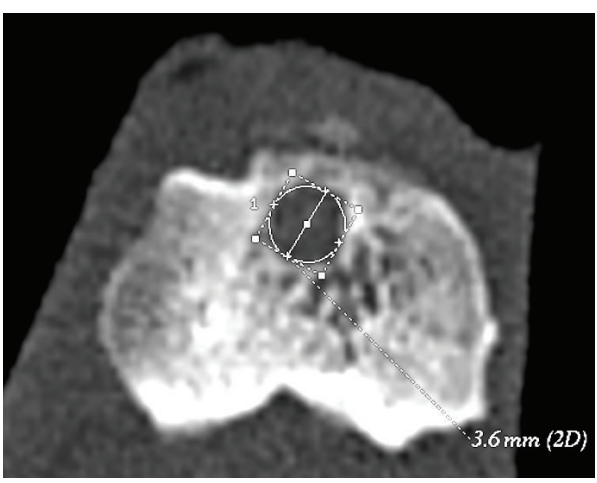

(d)

FIGURE 4: Femoral and tibial tunnels measured by CT scanning. Scanning plane confirmed in femora (a) and tibia (c). Minimum diameter of bone tunnel was measured in cross-section of femoral (b) or tibial (d) tunnel. Spacing between cross-sections was $0.5 \mathrm{~mm}$.

ligament to bone in clinic [29], while at insertion sites of BIO reconstruction groups, chondrocytes and collagen type II were found indicating a process of endochondral ossification.

Mechanical property is another key point in early healing of ACL reconstruction. After surgery the ligament bone healing process needs more than a year in clinic. During this time, patients are not allowed to participate in strenuous exercise. And the mechanical properties during this time are important for prospective knee joint stability. In our study, at 3 months after surgery, mechanical properties of substitution complex were significantly different among 3 groups. Failure load of complexes in both PLLA group and BIO group was significantly higher than that in control group. But only in BIO group, all implantations were broken from the middle of tendons, while in other two groups there were specimens pulling out of bone tunnel. These results showed stronger early stage healing of ligament to bone using autologous tendon and PLLA scaffold with E7 peptide conjugation transplantation. In rabbit, the reported maximum ACL force is about $138.6 \%$ of body weight [30]. According to this data, the maximum native ACL force in this study is about $40 \mathrm{~N}$ (rabbit weight: $2.5-3.0 \mathrm{~kg}$ ). The average failure load of control group was under $40 \mathrm{~N}$. And the PLLA and BIO scaffold groups were higher than it, $82.59 \pm 3.33$ and $141.50 \pm 3.90 \mathrm{~N}$, respectively.
Results of bone tunnel measurement showed no significant statistical differences in PLLA group and BIO group at 1 and 3 months. Only in control group there was significant enlargement of bone tunnel. But the diameter was smaller in $\mathrm{BIO}$ scaffolds implantation groups than that in control group. Both results indicated that composite scaffold of latticelike nanofibrous meshes can promote early stage healing of ligament-bone insertion site after ACL reconstruction of rabbit by narrowing bone tunnel and accelerating collagen structure remodeling to form direct junction.

\section{Conclusions}

In the present study, a substitution complex was designed by incorporating electrospinning nanofibrous meshes into autologous tendon. By modification of nanotopographical properties to lattice-like, the scaffold was suitable for MSCs proliferation, osteogenic differentiation, and active function. To absorb native MSCs from bone marrow, MSCs specific affinity peptide E7 was conjugated to nanofibrous meshes. After implantation of grafts for 3 months, the regenerated ligament-bone insertion exhibited abundant ECM (collagen I, II, and III) and fibrocartilage growth. The tensile strength of substitution complex was shown to be better than control. The findings of this study imply that nanofibrous scaffold with specific MSCs affinity peptide has great potentials 


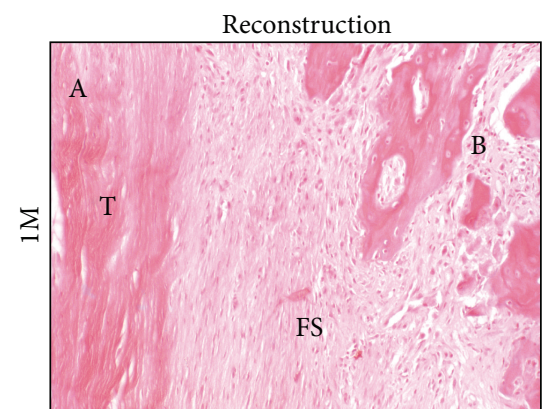

(a)

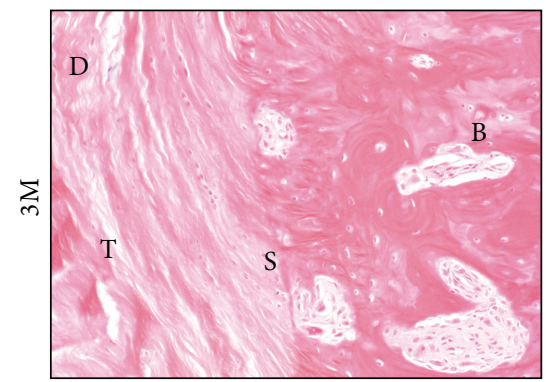

(d)

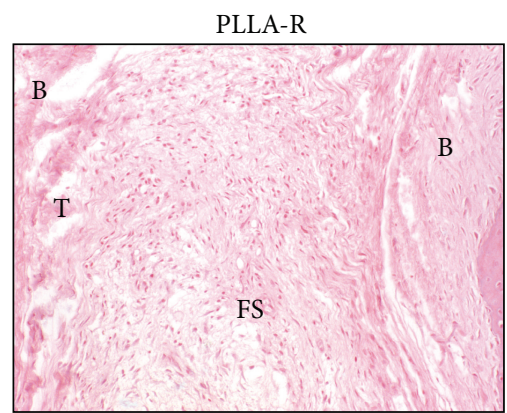

(b)

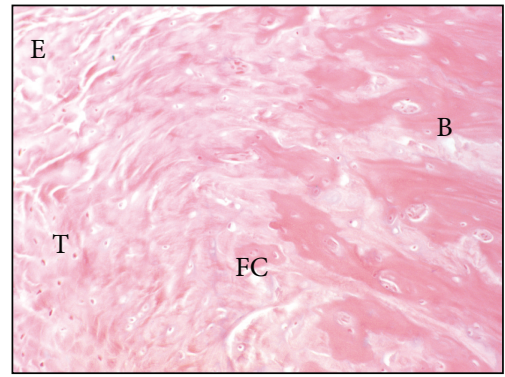

(e)

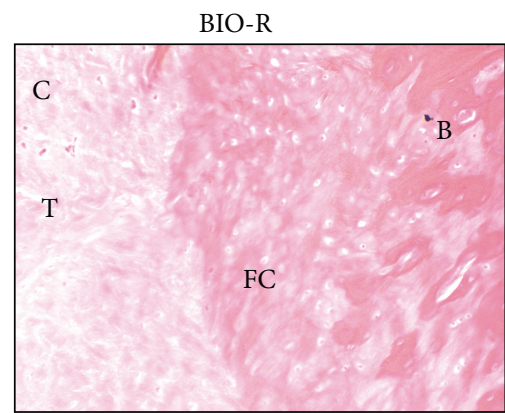

(c)

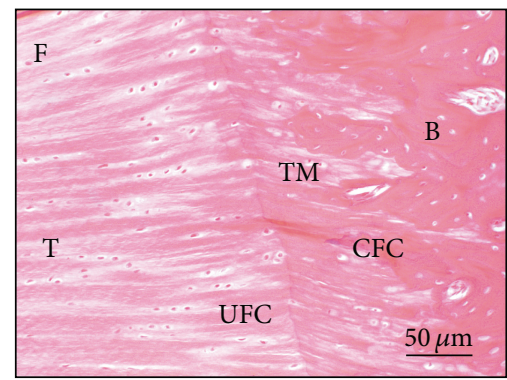

(f)

FIGURE 5: Histological assessment of ligament-bone insertion at 1 and 3 months after reconstruction surgery. H-E staining indicated connective tissues growing in ligament-bone insertion of control group, PLLA reconstruction group, and BIO reconstruction group at 1 month ((a)-(c)) and 3 months ((d)-(f)). Magnification: 200x. T: tendon; B: bone; FS: fibrous synovium; FC: fibrocartilage; S: Sharpey's fiber; UFC: uncalcified fibrocartilage; CFC: calcified fibrocartilage; TM: tide line mark.

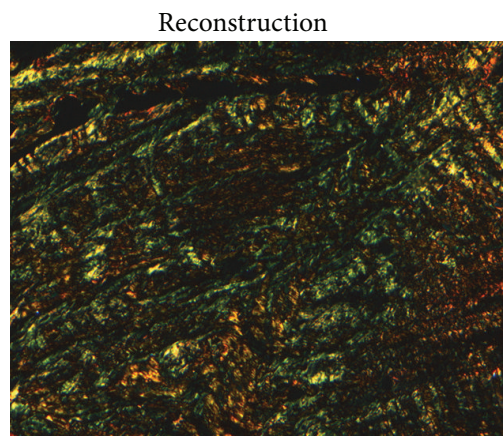

(a)

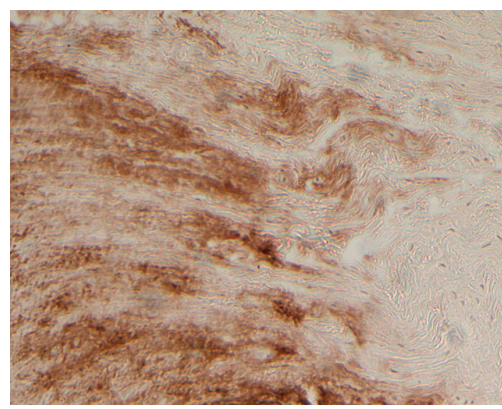

(d)

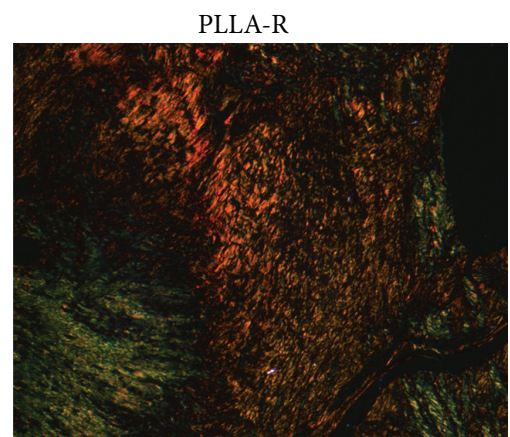

(b)

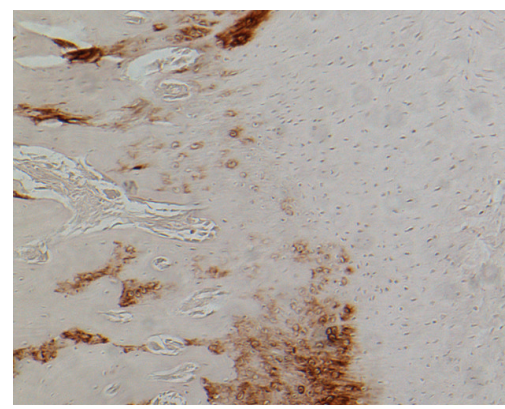

(e)

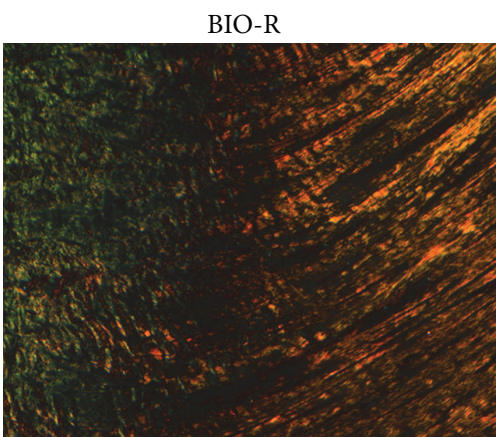

(c)

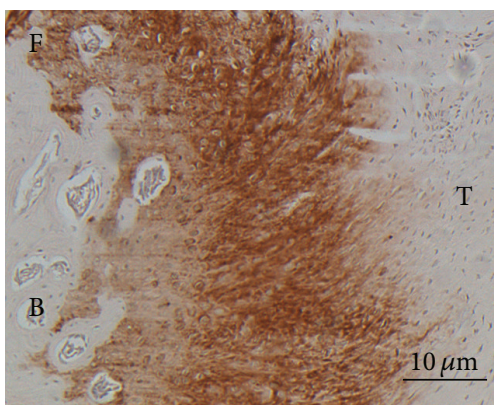

(f)

FIGURE 6: Sirius red and immunohistochemical staining of ligament-bone insertion at 3 months after ACL reconstruction surgery. Sirius red staining observed by polarized light microscope indicated distribution of collagen type I (red) and III (green) of control group, PLLA reconstruction group, and BIO reconstruction group $((\mathrm{a})-(\mathrm{c}))$. Specific immunohistochemical staining showing distribution of collagen type II at ligament-bone insertion in 3 groups ((d)-(f)). Magnification: 100x. T: tendon; B: bone. 
in accelerating ligament-bone healing process after ACL reconstruction.

\section{Conflict of Interests}

All authors declare that there is no conflict of interests regarding the publication of this paper.

\section{Acknowledgments}

This work was supported by the National Natural Science Foundation of China (Grant nos. 81171726, 81101390), the National Basic Research Program of China (2012CB933903), and the China Postdoctoral Science Foundation.

\section{References}

[1] M. M. Stevens and J. H. George, "Exploring and engineering the cell surface interface," Science, vol. 310, no. 5751, pp. 1135-1138, 2005.

[2] Y. Z. Zhang, B. Su, J. Venugopal, S. Ramakrishna, and C. T. Lim, "Biomimetic and bioactive nanofibrous scaffolds from electrospun composite nanofibers," International Journal of Nanomedicine, vol. 2, no. 4, pp. 623-638, 2007.

[3] C. K. Kuo, J. E. Marturano, and R. S. Tuan, "Novel strategies in tendon and ligament tissue engineering: advanced biomaterials and regeneration motifs," Sports Medicine, Arthroscopy, Rehabilitation, Therapy and Technology, vol. 2, article 20, 2010.

[4] S. Sahoo, J. G. Cho-Hong, and T. Siew-Lok, "Development of hybrid polymer scaffolds for potential applications in ligament and tendon tissue engineering," Biomedical Materials, vol. 2, no. 3, article 001, pp. 169-173, 2007.

[5] K. R. Reinhardt, I. Hetsroni, and R. G. Marx, "Graft selection for anterior cruciate ligament reconstruction: a level I systematic review comparing failure rates and functional outcomes," Orthopedic Clinics of North America, vol. 41, no. 2, pp. 249-262, 2010.

[6] F. A. Petrigliano, D. R. McAllister, and B. M. Wu, "Tissue engineering for anterior cruciate ligament reconstruction: a review of current strategies," Arthroscopy, vol. 22, no. 4, pp. 441451, 2006

[7] E. M. Horwitz, K. Le Blanc, M. Dominici et al., "Clarification of the nomenclature for MSC: the International Society for Cellular Therapy position statement," Cytotherapy, vol. 7, no. 5, pp. 393-395, 2005.

[8] M. F. Pittenger, A. M. Mackay, S. C. Beck et al., "Multilineage potential of adult human mesenchymal stem cells," Science, vol. 284, no. 5411, pp. 143-147, 1999.

[9] A. I. Caplan and D. Correa, “The MSC: an injury drugstore," Cell Stem Cell, vol. 9, no. 1, pp. 11-15, 2011.

[10] J. M. Karp and G. S. Leng Teo, "Mesenchymal stem cell homing: the devil is in the details," Cell Stem Cell, vol. 4, no. 3, pp. 206216, 2009.

[11] Z. Shao, X. Zhang, Y. Pi et al., "Polycaprolactone electrospun mesh conjugated with an MSC affinity peptide for MSC homing in vivo," Biomaterials, vol. 33, no. 12, pp. 3375-3387, 2012.

[12] J. Buschmann, L. Harter, S. Gao et al., "Tissue engineered bone grafts based on biomimetic nanocomposite PLGA/amorphous calcium phosphate scaffold and human adipose-derived stem cells," Injury, vol. 43, no. 10, pp. 1689-1697, 2012.
[13] N. Hild, O. D. Schneider, D. Mohn et al., "Two-layer membranes of calcium phosphate/collagen/PLGA nanofibres: in vitro biomineralisation and osteogenic differentiation of human mesenchymal stem cells," Nanoscale, vol. 3, no. 2, pp. 401-409, 2011.

[14] M. D. Schofer, P. P. Roessler, J. Schaefer et al., "Electrospun plla nanofiber scaffolds and their use in combination with bmp-2 for reconstruction of bone defects," PLoS ONE, vol. 6, no. 9, Article ID e25462, 2011.

[15] X. H. Zou, Y. L. Zhi, X. Chen et al., "Mesenchymal stem cell seeded knitted silk sling for the treatment of stress urinary incontinence," Biomaterials, vol. 31, no. 18, pp. 4872-4879, 2010.

[16] M. J. Dalby, N. Gadegaard, R. Tare et al., "The control of human mesenchymal cell differentiation using nanoscale symmetry and disorder," Nature Materials, vol. 6, no. 12, pp. 997-1003, 2007.

[17] J. Zhu, Q. Cai, X. Zhang et al., "Biological characteristics of mesenchymal stem cells grown on different topographical nanofibrous poly-L-lactide meshes," Journal of Biomedical Nanotechnology, vol. 9, no. 10, pp. 1757-1767, 2013.

[18] Y. Pi, X. Zhang, J. Shi et al., "Targeted delivery of non-viral vectors to cartilage in vivo using a chondrocyte-homing peptide identified by phage display," Biomaterials, vol. 32 , no. 26 , pp. 6324-6332, 2011.

[19] C. B. Ma, S. Kawamura, X.-H. Deng et al., "Bone morphogenetic proteins-signaling plays a role in tendon-to-bone healing: a study of rhBMP-2 and noggin," The American Journal of Sports Medicine, vol. 35, no. 4, pp. 597-604, 2007.

[20] C. N. Manning, H. M. Kim, S. Sakiyama-Elbert, L. M. Galatz, N. Havlioglu, and S. Thomopoulos, "Sustained delivery of transforming growth factor beta three enhances tendon-tobone healing in a rat model," Journal of Orthopaedic Research, vol. 29, no. 7, pp. 1099-1105, 2011.

[21] X. Huangfu and J. Zhao, “Tendon-bone healing enhancement using injectable tricalcium phosphate in a dog anterior cruciate ligament Reconstruction Model," Arthroscopy, vol. 23, no. 5, pp. 455-462, 2007.

[22] A. Bedi, D. Kovacevic, C. Hettrich et al., "The effect of matrix metalloproteinase inhibition on tendon-to-bone healing in a rotator cuff repair model," Journal of Shoulder and Elbow Surgery, vol. 19, no. 3, pp. 384-391, 2010.

[23] L. V. Gulotta, D. Kovacevic, J. D. Packer, X. H. Deng, and S. A. Rodeo, "Bone marrow-derived mesenchymal stem cells transduced with scleraxis improve rotator cuff healing in a rat model," The American Journal of Sports Medicine, vol. 39, no. 6, pp. 1282-1289, 2011.

[24] K. von der Mark, J. Park, S. Bauer, and P. Schmuki, "Nanoscale engineering of biomimetic surfaces: cues from the extracellular matrix," Cell and Tissue Research, vol. 339, no. 1, pp. 131-153, 2010.

[25] T. K. H. Teh, S.-L. Toh, and J. C. H. Goh, "Aligned hybrid silk scaffold for enhanced differentiation of mesenchymal stem cells into ligament fibroblasts," Tissue Engineering C, vol. 17, no. 6, pp. 687-703, 2011.

[26] Z. Yin, X. Chen, J. L. Chen et al., “The regulation of tendon stem cell differentiation by the alignment of nanofibers," Biomaterials, vol. 31, no. 8, pp. 2163-2175, 2010.

[27] B. M. Baker, A. S. Nathan, A. O. Gee, and R. L. Mauck, "The influence of an aligned nanofibrous topography on human mesenchymal stem cell fibrochondrogenesis," Biomaterials, vol. 31, no. 24, pp. 6190-6200, 2010. 
[28] A. Martins, M. L. Alves da Silva, S. Faria, A. P. Marques, R. L. Reis, and N. M. Neves, "The influence of patterned nanofiber meshes on human mesenchymal stem cell osteogenesis," Macromolecular Bioscience, vol. 11, no. 7, pp. 978-987, 2011.

[29] H. Robert, J. Es-Sayeh, D. Heymann, N. Passuti, S. Eloit, and E. Vaneenoge, "Hamstring insertion site healing after anterior cruciate ligament reconstruction in patients with symptomatic hardware or repeat rupture: a histologic study in 12 patients," Arthroscopy, vol. 19, no. 9, pp. 948-954, 2003.

[30] D. L. Gushue, J. Houck, and A. L. Lerner, "Rabbit knee joint biomechanics: motion analysis and modeling of forces during hopping," Journal of Orthopaedic Research, vol. 23, no. 4, pp. 735-742, 2005. 

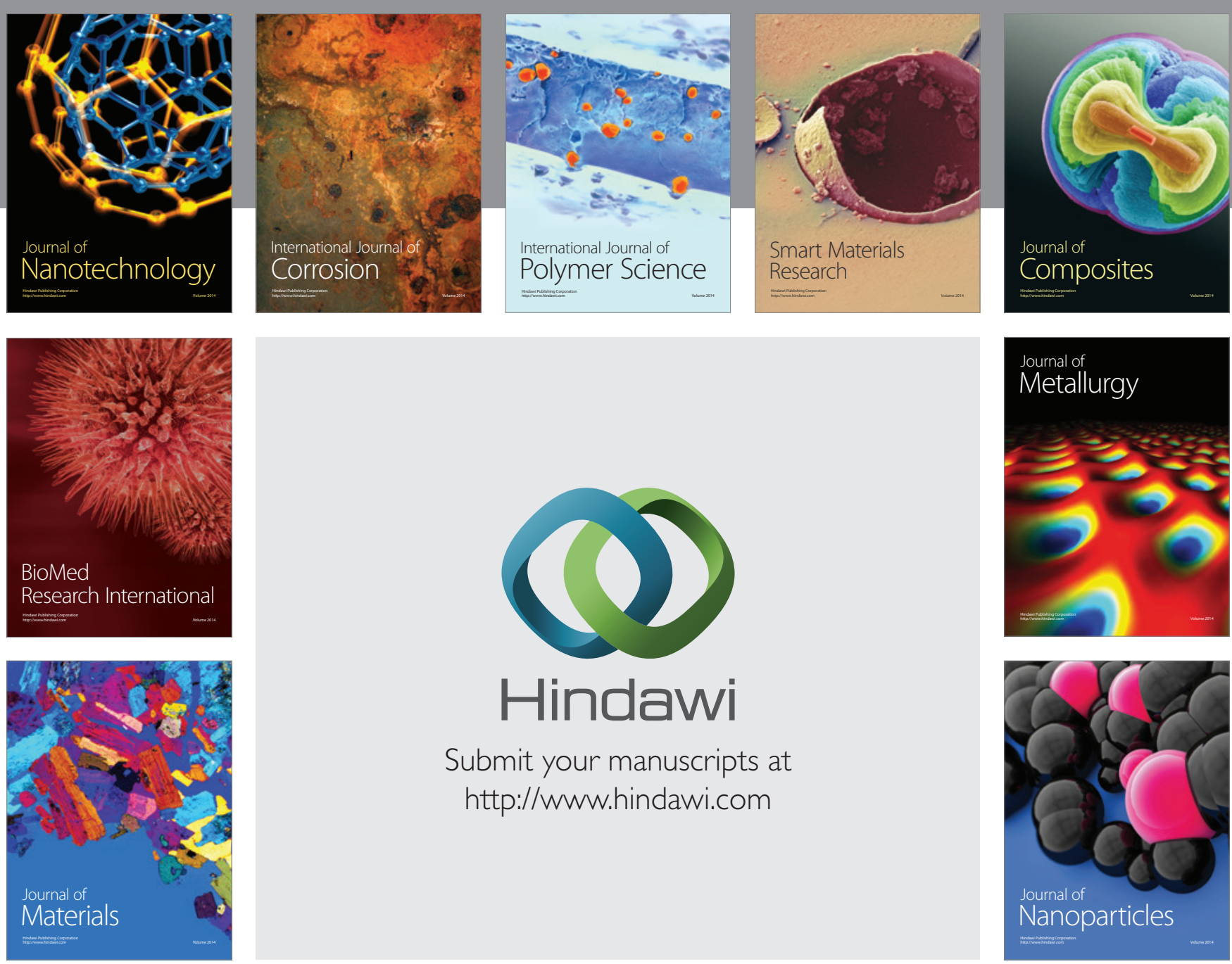

Submit your manuscripts at http://www.hindawi.com
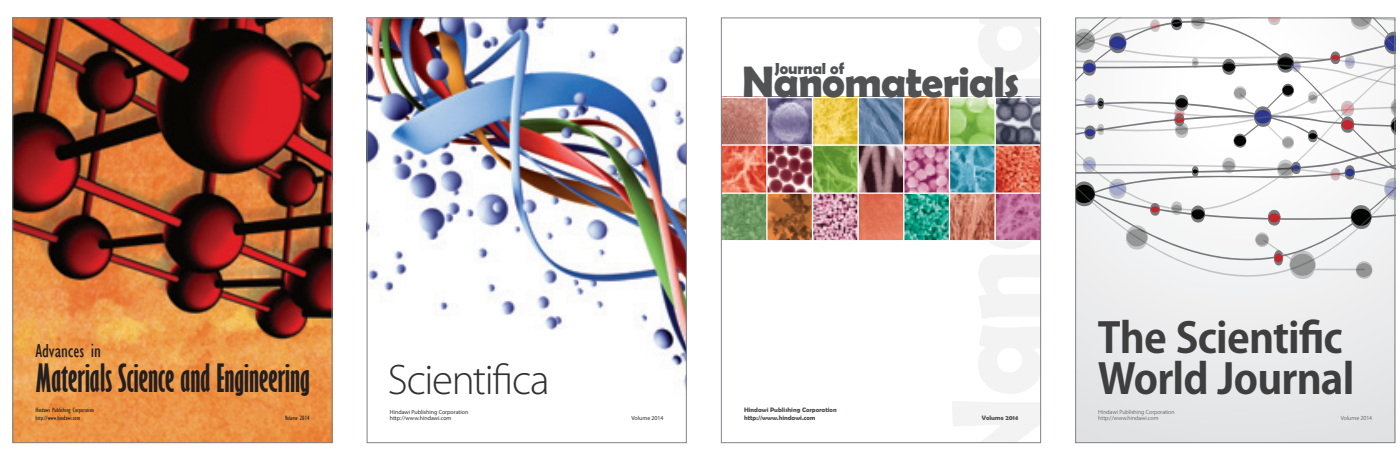

\section{The Scientific World Journal}
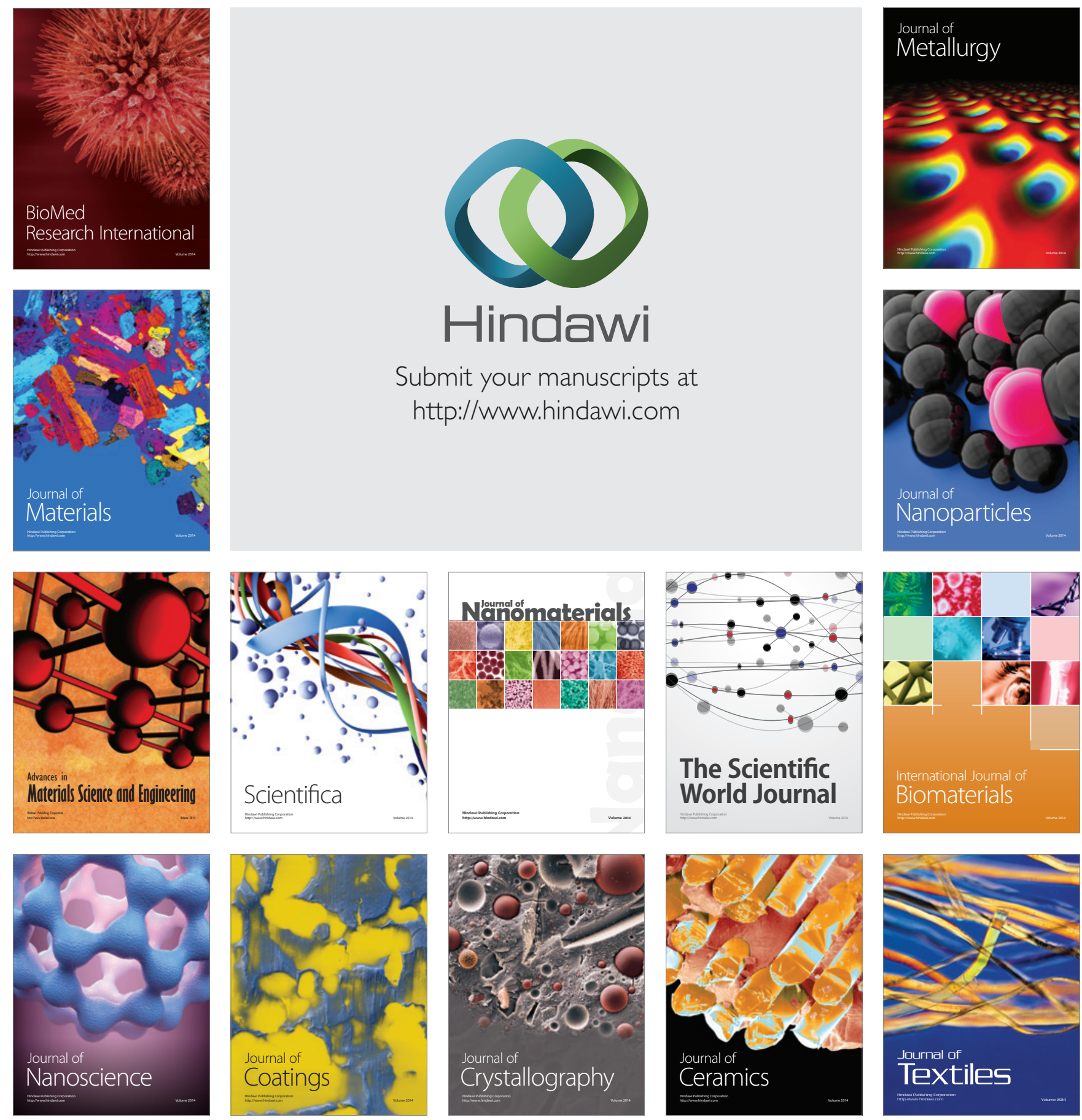